ARTIGO ORIGINAL

\title{
Produção e caracterização de blocos vazados de cimento-madeira de baixa densidade para construção civil
}

\author{
Production and evaluation of low-density cast-wood-cement blocks for civil \\ construction
}

\author{
Barbara Talamini Villas-Bôas ${ }^{1}$ (D), Setsuo Iwakiri ${ }^{1}$ (D), Carlos Frederico Alice Parchen $^{1}$ (D) \\ ${ }^{1}$ Universidade Federal do Paraná - UFPR, Curitiba, PR, Brasil
}

Como citar: Villas-Bôas, B. T., Iwakiri, S., \& Parchen, C. F. A. (2020). Produção e caracterização de blocos vazados de cimento-madeira de baixa densidade para construção civil. Scientia Forestalis, 48(126), e3177.

https://doi.org/10.18671/scifor.v48n126.22

\begin{abstract}
Resumo
Este trabalho teve como objetivo avaliar a viabilidade de uso de resíduos de madeira de Pinus spp provenientes da indústria madeireira, na forma de cavacos, para produção de blocos vazados de concreto simples de baixa densidade para aplicações em alvenarias internas. Foram utilizados cavacos passantes pela peneira de $4,75 \mathrm{~mm}$ e retidos na de $2,36 \mathrm{~mm}$, os quais foram previamente tratados em suspensão de $\mathrm{Ca}(\mathrm{OH})_{2}$. Os blocos foram produzidos com as relações madeira:cimento (traços) de 1:1,5 e 1:2,2 (CP II-Z) e 1:2,2 (CP V-ARI), utilizando processo de vibro-compactação. As propriedades avaliadas conforme recomendações da NBR 12118-2014 foram: teor de umidade, densidade aparente, absorção de água, resistência à compressão axial e condutividade térmica. Os resultados obtidos indicaram um aumento na densidade e resistência à compressão axial dos blocos com maiores quantidades de cimento, independentemente do tipo de cimento. Os resultados de condutividade térmica indicaram melhor isolamento térmico dos blocos de cimento-madeira em comparação aos materiais cimentícios convencionais. Os blocos apresentaram resistência à compressão axial menor que o requisito mínimo de 3,0 MPa (NBR 6136-2014) estabelecido para bloco estrutural. Entretanto, a sua produção pode ser recomendada para aplicações em alvenaria interna, sem função estrutural, com a substituição de agregado mineral por vegetal (madeira) com consequente redução no peso do produto.
\end{abstract}

Palavras-chave: Madeira de pinus; Resistência à compressão axial; Cavacos de madeira.

\begin{abstract}
The objective of this work was to evaluate the feasibility of the use of Pinus spp (Pinus elliotti and Pinus taeda) wood chips from the lumber industry for the production of simple low-density concrete cast blocks for internal brickwork applications. Chips previously treated in $\mathrm{Ca}(\mathrm{OH})_{2}$ suspension were classified by passing the $4.75 \mathrm{~mm}$ sieve and retaining in the $2.36 \mathrm{~mm}$ sieve, were used. The blocks were produced with wood:cement ratios (traces) of 1:1.5 and 1:2.2 (CP II-Z) and 1:2.2 (CP V-ARI), using the vibro-compaction process. The evaluated properties (NBR 12118-2014) were: moisture content, apparent density, water absorption, axial compression strength and thermal conductivity. The obtained results indicated an increase in density and resistance to axial compression of the blocks with higher ratios, regardless of the type of cement. The results of thermal conductivity indicated better thermal insulation of the blocks of wood:cement compared to conventional cementitious materials. The blocks presented axial compression strength below the minimum requirement of 3.0 MPa (NBR 6136-2014) established for structural blocks. However, its production can be recommended for applications in internal brickwork, without structural function, with the substitution of mineral aggregate by vegetable (wood), with consequent reduction in product weight.
\end{abstract}

Keywords: Wood of pinus; Axial compression strength; Wood chips.

Fonte de financiamento: Nenhuma.

Conflito de interesse: Nada a declarar.

Autor correspondente: setsuo.ufpr@gmail.com

Recebido: 29 outubro 2018

Aceito: 29 setembro 2019.

Editor: Francides Gomes da Silva Júnior.

(i) Este é um artigo publicado em acesso aberto (Open Access) sob a licença Creative Commons Attribution, que permite uso, distribuição e reprodução em qualquer meio, sem restrições desde que o trabalho original seja corretamente citado. 


\section{INTRODUÇÃO}

Nos últimos anos, tem crescido a atenção dada ao aquecimento global, fenômeno que se deve à liberação de gases causadores do efeito estufa. Apesar de existirem incertezas associadas à mudança de clima, a maioria da comunidade científica internacional reconhece que o fenômeno do aquecimento do planeta é fruto da atividade humana. Segundo o Instituto Carbono Brasil (2014), os principais gases causadores do efeito estufa são o $\mathrm{CO}_{2}$, o metano e o óxido nitroso.

De acordo com Torkaman et al. (2014), nos próximos anos, a indústria da construção civil terá o desafio de incorporar a sustentabilidade em seus processos de produção; entre as várias medidas, uma será a fabricação de produtos que contribuam para a redução de $\mathrm{CO}_{2}$ na atmosfera, incorporando resíduos de outras atividades industriais em seus processos.

O resíduo florestal é todo e qualquer material resultante da colheita ou do processamento da madeira ou outro recurso florestal que permaneça sem utilização definida ao longo do processo (Nolasco, 2000). Grande volume de resíduos de madeira é empilhado, permanecendo em diversos estágios de decomposição (Rech, 2002). No entanto, segundo Parchen (2012), começa a ocorrer uma mudança de rumo a esse respeito e o que se entendia como "entulho" (material inútil) passa a ter um novo aspecto de resíduo renovável.

Os produtos fabricados com madeira armazenam $\mathrm{CO}_{2}$, um exemplo desses produtos são os compósitos de cimento-madeira. Nesse caso, o cimento é utilizado como aglomerante mineral e misturado com a madeira, como agregado. Iwakiri (2005), Lima (2009) e Savastano Junior (2000) citaram como vantagens desse produto: o isolamento térmico e acústico, o alto grau de resistência ao fogo e a impactos, as ótimas características mecânicas além da resistência ao ataque de agentes xilófagos. Ainda nesse sentido, Semple \& Evans (2004) afirmaram que a baixa densidade desses compósitos permite sua utilização na produção de painéis de fechamento, forros, telhas e elementos de vedação; por outro lado, Lima (2009) indica a possibilidade de uso de resíduos de processamento de toras de Pinus spp como substituição ao agregado miúdo na produção de blocos de concreto para alvenaria estrutural.

A NBR 6136 (Associação Brasileira de Normas Técnicas, 2014a) define blocos vazados de concreto simples como um componente para execução de alvenaria, com ou sem função estrutural, vazado nas faces superior e inferior, cuja área líquida é igual ou inferior a 75\% da área bruta. Atualmente, há um novo conceito de blocos de concreto, mais leves que os tradicionais. De acordo com a NBR 12655 (Associação Brasileira de Normas Técnicas, 2015), o concreto pode ser classificado conforme sua densidade no estado endurecido; assim, o concreto denominado leve possui densidade não menor que $800 \mathrm{~kg} / \mathrm{m}^{3}$, mas não excede $2.000 \mathrm{~kg} / \mathrm{m}^{3}$.

Segundo Del Coz Díaz et al. (2009), o grande interesse da indústria da construção civil pelos blocos mais leves deve-se à sua baixa densidade e, especialmente, às propriedades de isolamento térmico. Uma vez que a maioria dos elementos estruturais é feita de concreto armado, sendo os painéis e paredes também fabricados com produtos à base de cimento Portland, existe uma constante preocupação com os problemas de tamanho e peso dos elementos. A fim de reduzir o peso das estruturas, podem-se utilizar blocos leves de concreto, tornando as estruturas mais leves e diminuindo o custo de construção (Posi et al., 2013). Dargahi-Zaboli \& Alizadeh (2014) destacam que, além de reduzir o peso da estrutura, esses blocos possuem outras vantagens, como resistência ao fogo e maior resistência contra intempéries (como o clima frio).

Há anos, os concretos leves têm sido investigados, principalmente para utilização em paredes de alvenaria, pois apresentam elevada relação resistência: peso, baixo coeficiente de condutibilidade térmica e boa característica de isolamento acústico. Basicamente, os materiais utilizados para sua fabricação são: aglomerantes (usualmente, cimento Portland), agregados graúdos e miúdos e água (Posi et al., 2013).

Os equipamentos utilizados para sua produção são chamados vibro-compactadoras (prensas), devido ao mecanismo empregado para fazer com que o material penetre e preencha os moldes (Medeiros \& Sabbatini, 1993). Segundo Sousa (2001), o responsável pelo 
preenchimento e adensamento da mistura é o sistema de vibração e o mecanismo responsável pelo adensamento e controle da altura dos blocos é o sistema de compressão. Nesse processo, a sequência de colocação dos materiais e o tempo adequado de mistura devem ser observados, de maneira a ser compatíveis com a produtividade do equipamento. Ainda, para garantir que o produto final obtenha o grau de compactação necessário, devem-se respeitar os tempos de alimentação e vibração (Medeiros \& Sabbatini, 1993).

Após a moldagem, os blocos são transportados até o local de cura, sendo este um conjunto de medidas que visam a proporcionar aos blocos as condições de umidade, temperatura e pressão necessárias a uma adequada reação de hidratação do cimento, uma vez que as condições de umidade e temperatura, principalmente nos primeiros dias, têm muita importância nas propriedades do concreto endurecido (Tango, 1984).

Perante o exposto, o objetivo desta pesquisa foi avaliar as propriedades físicas, mecânicas e térmicas de blocos vazados de concreto simples, de baixa densidade, produzidos com resíduos de madeira de Pinus spp e cimento, com diferentes relações madeira: cimento (traços).

\section{MATERIAL E MÉTODOS}

\section{Material}

A madeira de Pinus spp (preponderantemente Pinus elliottii e Pinus taeda) em forma de cavacos, utilizada nesta pesquisa, foi procedente de uma madeireira localizada no município de Tunas do Paraná, Estado do Paraná. Como aglomerante para produção dos blocos foram utilizados dois tipos de cimentos Portland: CP II-Z e CP V-ARI.

O CP II-Z possui adição de material pozolânico, comercializado facilmente em lojas de materiais de construção, com custo não muito alto, pode ser utilizado na produção de artefatos de cimento, sendo o concreto feito com ele mais impermeável. O CP V-ARI é adequado para aplicações onde necessita-se de elevada resistência às primeiras idades. O desenvolvimento prematuro de resistência é conseguido graças à utilização de uma dosagem diferenciada de calcário e argila na produção do clínquer, e pela moagem mais fina do cimento (Associação Brasileira de Cimento Portland, 2013).

Além de aglomerante aéreo para o pré-tratamento das partículas, realizado com suspensão de hidróxido de cálcio - $\mathrm{Ca}(\mathrm{OH})_{2}$, preparada por meio de mistura de cal $(\mathrm{CaO})$ e água $\left(\mathrm{H}_{2} \mathrm{O}\right)$.

\section{Fabricação dos blocos}

Inicialmente, os cavacos foram secos em ambiente natural ao teor de umidade médio de $15 \%$ e processados no moinho de martelos, utilizando-se uma peneira de 1" (2,54 cm). Após a moagem, o material foi classificado em peneiras, sendo que, as partículas passantes na peneira de $4,75 \mathrm{~mm}$ e retidas na de $2,36 \mathrm{~mm}$, foram utilizadas na produção de blocos vazados de alvenaria.

Para a fabricação de blocos foram utilizados cimento, partículas de madeira, hidróxido de cálcio - $\mathrm{Ca}(\mathrm{OH})_{2}$ e água nas quantidades apresentadas no planejamento experimental da Tabela 1.

Tabela 1. Planejamento experimental.

\begin{tabular}{|c|c|c|c|}
\hline \multirow{2}{*}{ Material } & Bloco tipo A & Bloco Tipo B & Bloco Tipo C \\
\hline & \multicolumn{3}{|c|}{ 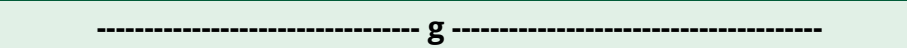 } \\
\hline Cimento & 2.140 & 2.944 & 2.944 \\
\hline Partículas & 1.640 & 1.539 & 1.539 \\
\hline $\mathrm{Ca}(\mathrm{OH})_{2}$ & 1.640 & 1.539 & 1.539 \\
\hline Água & 642 & 883 & 883 \\
\hline
\end{tabular}

Bloco A: cimento CP II-Z, relação madeira: cimento de 1:1,5; Bloco B: cimento CP II-Z, relação madeira: cimento de 1:2,2; Bloco A: cimento CP V ARI, relação madeira: cimento de 1:2,2. 
O cimento Portland tipo CP II-Z foi utilizado para produção de blocos com relação madeira:cimento (traços) de 1:1,5 (tipo A) e de 1:2,2 (tipo B), e o cimento Portland CP-V ARI com a relação madeira:cimento de 1:2,2 (tipo C). Estes traços foram escolhidos a partir do estudo de Parchen (2012). A quantidade de água foi de 30\% em relação à massa do cimento, e a de hidróxido de cálcio $\mathrm{Ca}(\mathrm{OH})_{2}$ foi definida conforme recomendações de Villas-Bôas et al. (2017), que indicaram uma relação água $\left(\mathrm{H}_{2} \mathrm{O}\right)$ : $\mathrm{Cal}(\mathrm{CaO})$ igual a 6,25:1. Foram fabricados nove blocos para cada tipo (A, B e C), com as dimensões externas de $140 \times 190 \times 390 \mathrm{~mm}$ (largura $\times$ altura $\times$ comprimento), totalizando 27 blocos, este valor foi escolhido devido ao modelo da vibro-compactadora que produzia três blocos por vez e a quantidade de resíduo fornecido pela madeireira.

A mistura dos materiais foi realizada em um misturador com tambor fixo e eixo horizontal com capacidade de 200 litros (seco), e o tempo de mistura foi de 120 a 150 segundos (Figura 1).

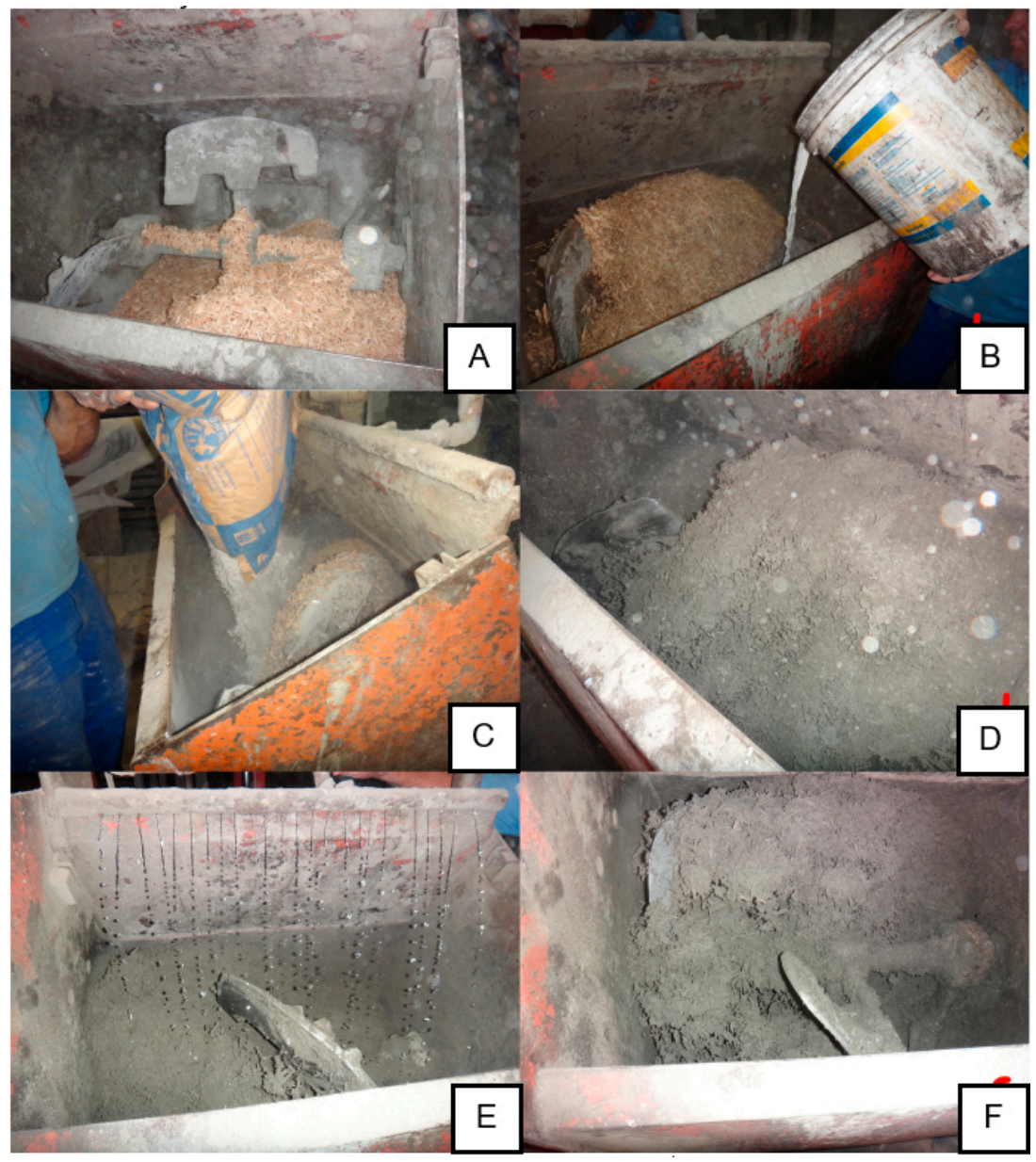

Figura 1. Mistura de materiais para produção dos blocos de cimento-madeira vazados. (A) partículas de madeira inseridas no misturador; (B) adição da suspensão de $\mathrm{Ca}(\mathrm{OH})_{2} ;(\mathrm{C})$ adição do cimento Portland;

(D) mistura dos três materiais; (E) adição de água; (F) mistura pronta para produção dos blocos.

Para a produção dos blocos, foi utilizada uma prensa vibro-compactadora da marca Trillor Montana, modelo MBX 200, sendo que a sapata aplicava uma pressão de 0,05 MPa, distribuída durante a compressão do concreto em estado fresco para produção de três blocos de cada vez. Essa máquina é considerada simplificada, de baixo custo e de grande praticidade. Não possui silo para o concreto, nem gaveta alimentadora, sendo o enchimento da forma por processo manual, a partir da mesa posicionada na parte traseira da máquina, que também é abastecida manualmente (Figura 2). 


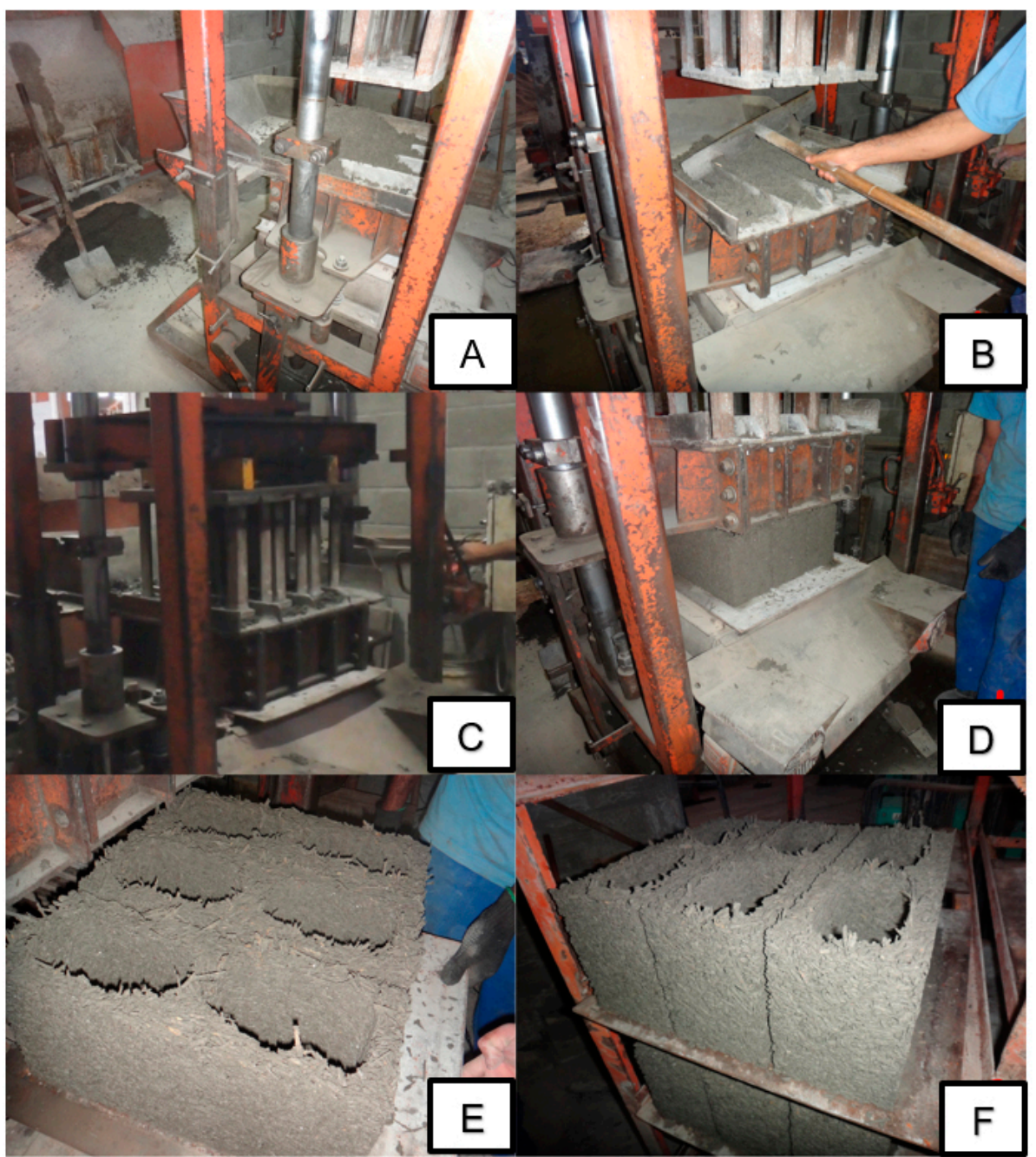

Figura 2. Produção de blocos de cimento-madeira vazados na prensa vibro-compactadora. (A) retirada e transferência do material do misturador da prensa vibro-compactadora; (B) material sendo vibrado e espalhado dentro das formas; (C) material sendo compactado pelas sapatas; (D) levantamento das formas; (E) retirada dos blocos da prensa; (F) blocos aguardando a cura.

Após a mistura dos materiais, a prensa vibro-compactadora foi abastecida com a quantidade requerida para confecção de três blocos. $O$ abastecimento das formas foi realizado em três camadas iguais, cada uma foi vibrada por 15 segundos, totalizando 45 segundos no processo. Após o enchimento das formas, as sapatas da prensa vibro-compactadora foram abaixadas para compactação e vibração final das peças. O tempo de vibro-compressão até a peça atingir a altura desejada também foi de 15 segundos.

Ao término da vibro-compactação, os blocos formados foram retirados manualmente da máquina, reiniciando-se outro ciclo. Após a retirada dos blocos da máquina, os mesmos foram encaminhados para a cura nas condições ambientais ao ar livre, em local coberto e protegido da insolação direta.

\section{Ensaios físicos, mecânico e térmico dos blocos}

Após o acondicionamento, nove blocos inteiros foram separados para determinação das propriedades físicas: teor de umidade, densidade aparente e absorção de água, de acordo com a norma NBR 6136 (Associação Brasileira de Normas Técnicas, 2014a), especifica para blocos vazados de concreto. Os ensaios seguem a metodologia indicada na NBR 12118 (Associação Brasileira de Normas Técnicas, 2014b). A resistência à compressão axial foi determinada para nove blocos inteiros, sendo três para cada tratamento. 
Para a determinação da condutividade térmica dos blocos, foram retirados das paredes dos blocos quatro prismas de $100 \times 100 \mathrm{~mm}$, os quais foram lixados de forma a favorecer a leitura no condutivímetro. Os ensaios foram realizados no aparelho condutivímetro-fluxímetro, ilustrado na Figura 3, e conduzidos com base nos procedimentos descritos na norma ASTM C-518 (American Society for Testing and Materials, 2010).

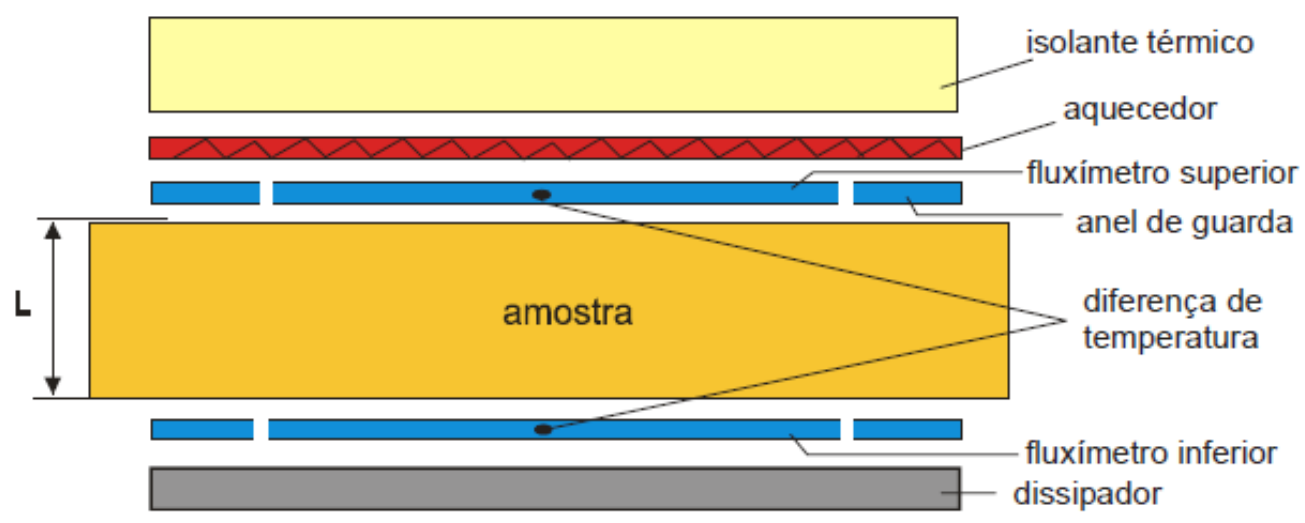

Figura 3. Esquema ilustrativo do condutivímetro-fluxímetro.

Os resultados dos ensaios foram submetidos à análise de variância (ANOVA) e ao teste de Tukey para comparação de médias, ao nível de probabilidade de 95\%. A ferramenta estatística utilizada para a análise de dados foi o software Statgraphics Centurion 16.2.

\section{RESUTADOS E DISCUSSÕES}

Os blocos vazados de cimento-madeira de baixa densidade produzidos pelo processo de vibro-compactação estão ilustrados na Figura 4.

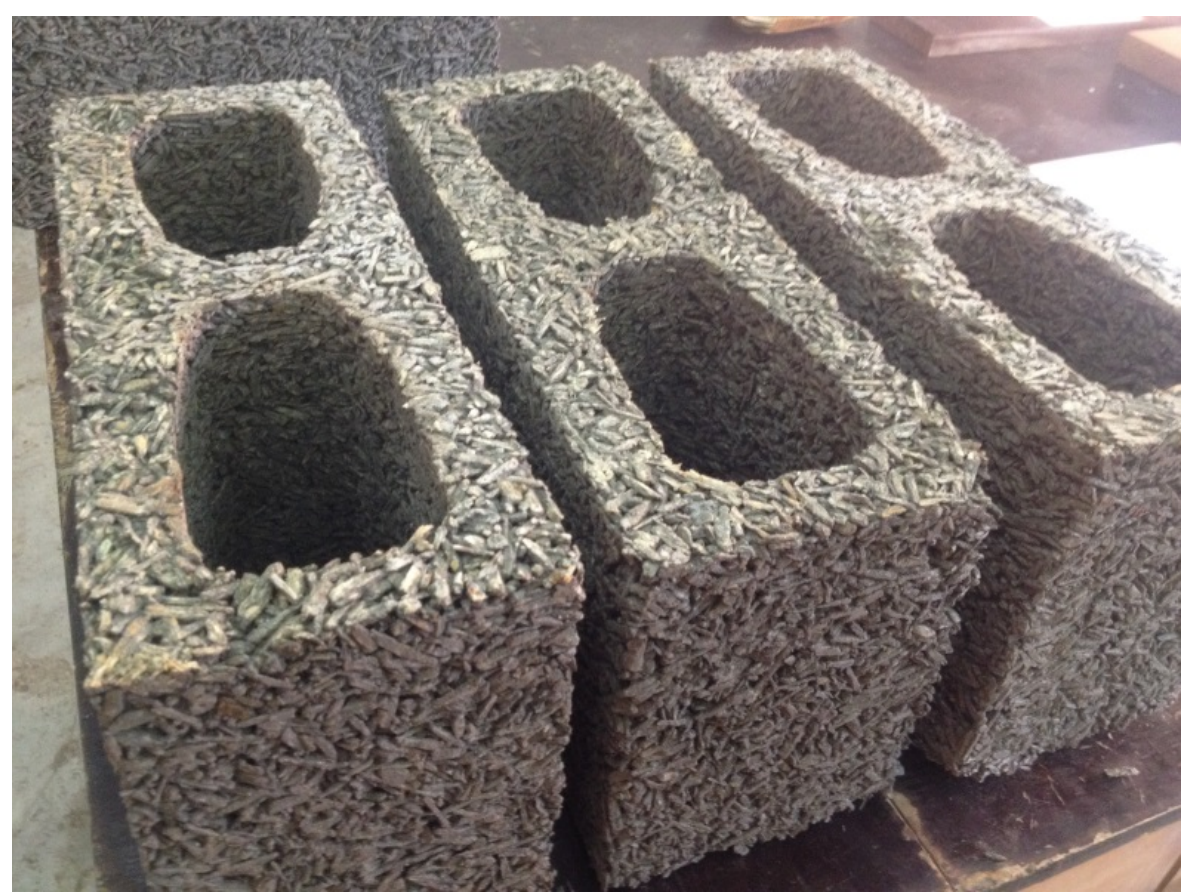

Figura 4. Blocos vazados de cimento-madeira de baixa densidade produzidos. 


\section{Propriedades físicas dos blocos}

Na Tabela 2 estão apresentados os valores médios do teor de umidade, densidade aparente e absorção de água ( 24 horas) para os três tipos de blocos produzidos.

Tabela 2. Propriedades físicas dos blocos de cimento-madeira vazados.

\begin{tabular}{cccc}
\hline Bloco & Umidade (\%) & $\begin{array}{c}\text { Densidade Aparente } \\
\left(\mathbf{g} / \mathbf{c m}^{\mathbf{3}} \mathbf{)}\right.\end{array}$ & Absorção de água (\%) \\
\hline A & $31,43 \mathrm{~b}$ & $0,54(0,037) \mathrm{b}$ & $48,24 \mathrm{a}$ \\
B & $33,94 \mathrm{a}$ & $0,72(0,021) \mathrm{a}$ & $42,85 \mathrm{~b}$ \\
C & $34,98 \mathrm{a}$ & $0,69(0,044) \mathrm{a}$ & $36,22 \mathrm{c}$ \\
\hline
\end{tabular}

Médias seguidas de mesmas letras nas colunas não diferem estatisticamente pelo teste de Tukey a 5\% de significância; valores entre parênteses se referem ao desvio padrão.

Os teores de umidade médio dos blocos foram de 31,43\%, 33,94\% e 34,98\%, respectivamente, para os tipos - A, B e C. Para o cálculo do teor de umidade a norma NBR 12118 (Associação Brasileira de Normas Técnicas, 2014b) indica a utilização da massa saturada dos corpos de prova, por esta razão os valores resultaram altos. Os blocos tipo A apresentaram valor médio de teor de umidade estatisticamente inferior em relação aos blocos B e C. Embora as diferenças sejam pequenas em termos de valores absolutos, o menor teor de umidade dos blocos $A$, pode ser atribuído à menor quantidade de água na sua composição em comparação aos tipos B e C, conforme as quantidades de materiais apresentados na Tabela 1.

Os valores médios de densidade dos blocos foram respectivamente de $0,54 \mathrm{~g} / \mathrm{cm}^{3}$ (tipo A), 0,72 $\mathrm{g} / \mathrm{cm}^{3}$ (tipo B) e $0,69 \mathrm{~g} / \mathrm{cm}^{3}$ (tipo C). Os blocos produzidos com relação madeira:cimento de 1;1,5 (tipo $A$ ) apresentaram valor médio de densidade estatisticamente inferior em relação aos blocos B e C, produzidos com relação madeira:cimento de 1: 2,2. Devido à maior densidade do cimento em comparação à madeira, o aumento na proporção de cimento na composição dos blocos elevou a sua densidade. Não foram constatadas diferenças significativas entre as densidades dos blocos produzidos com diferentes tipos de cimento - CP II-Z e CP V ARI.

Os valores médios de densidades obtidos neste estudo $\left(0,54 \mathrm{~g} / \mathrm{cm}^{3}\right.$ a $\left.0,72 \mathrm{~g} / \mathrm{cm}^{3}\right)$ estão próximos aos valores dos corpos de prova obtidos por Parchen (2012), de 0,64 e 0,74 g/cm para compósitos produzidos com relação madeira:cimento de 1:2,2 utilizando processo de vibro-compressão. Estas densidades correspondem à classificação dos blocos leves de concreto, conforme a norma NBR 12655 (Associação Brasileira de Normas Técnicas, 2015).

Com relação à absorção de água, os valores médios obtidos para os blocos tipos - A, B e C foram respectivamente de $48,24 \%, 42,85 \%$ e $36,22 \%$, sendo as diferenças estatisticamente significativas entre si. $O$ valor médio de absorção de água do bloco $A$, foi estatisticamente superior em relação ao bloco $B$, e este, em relação ao bloco $C$.

Maior absorção de água obtido para os blocos do tipo $\mathrm{A}$, pode ser atribuída à baixa quantidade de cimento, não ocorrendo a total encapsulação das partículas de madeira, com consequente aumento da penetração de água. Segundo Parchen et al. (2014), altos valores de absorção de água dos compósitos cimento-madeira são indicativos de sua aplicação restrita ao fechamento interno das construções.

\section{Resistência à compressão axial dos blocos}

Na Tabela 3 estão apresentados os valores médios da resistência à compressão axial para os três tipos de blocos produzidos. 
Tabela 3. Resistência à compressão axial dos blocos.

\begin{tabular}{cc}
\hline Bloco & Resistência à compressão axial (MPa) \\
\hline A & $1,09(0,041) b$ \\
B & $1,26(0,066) a b$ \\
C & $1.42(0,099) a$ \\
\hline
\end{tabular}

Médias seguidas de mesmas letras na coluna não diferem estatisticamente pelo teste de Tukey a 5\% de significância; valores entre parêntesis se referem ao desvio padrão.

Os valores médios de resistência axial dos blocos variaram de 1,09 MPa (tipo A) a 1,42 MPa (tipo C). Estes resultados estão próximos aos encontrados por Stancato (2006) para blocos moldados em fábrica, cujos valores de resistência à compressão axial variaram de 1,43 MPa a 1,72 MPa.

Os blocos do tipo $C$, produzidos com a relação madeira:cimento de 1:2.2 apresentaram média estatisticamente igual em comparação aos blocos do tipo $B$, com relação 1:2,2 e, superior aos blocos do tipo A, com relação madeira:cimento de 1:1,5.

A maior quantidade de cimento na composição dos blocos B e C contribuiu para o aumento da resistência axial. Castro et al. (2018) constataram também um aumento na resistência axial dos compósitos com aumento da relação madeira:cimento de 1:1,5 para 1:2 e 1:2,75. Já, Iwakiri et al. (2017) não observaram diferenças significativas entre os compósitos produzidos com relação madeira:cimento de 1:2,5 e 1:3. Portanto, o aumento na proporção de cimento a partir de uma determinada quantidade não afetam significativamente a resistência mecânica dos compósitos cimento-madeira.

Com relação aos tipos de cimento, não foram constatadas diferenças significativas entre os blocos produzidos com cimento Portland CP-II Z (tipo B) e cimento CP V ARI (tipo C), para mesma relação madeira:cimento de 1:2,2.

Os valores de resistência axial obtidos nesta pesquisa foram abaixo do valor mínimo recomendado pela norma NBR 6136 (Associação Brasileira de Normas Técnicas, 2014a) de 3,0 MPa, para uso estrutural portanto a sua aplicação deve ser recomendada para fechamento interno das construções.

No gráfico da Figura 5 estão apresentadas as comparações entre os valores médios de resistência axial considerando-se a área bruta, condições empregadas neste estudo e para a área líquida, para simulação de blocos maciços.

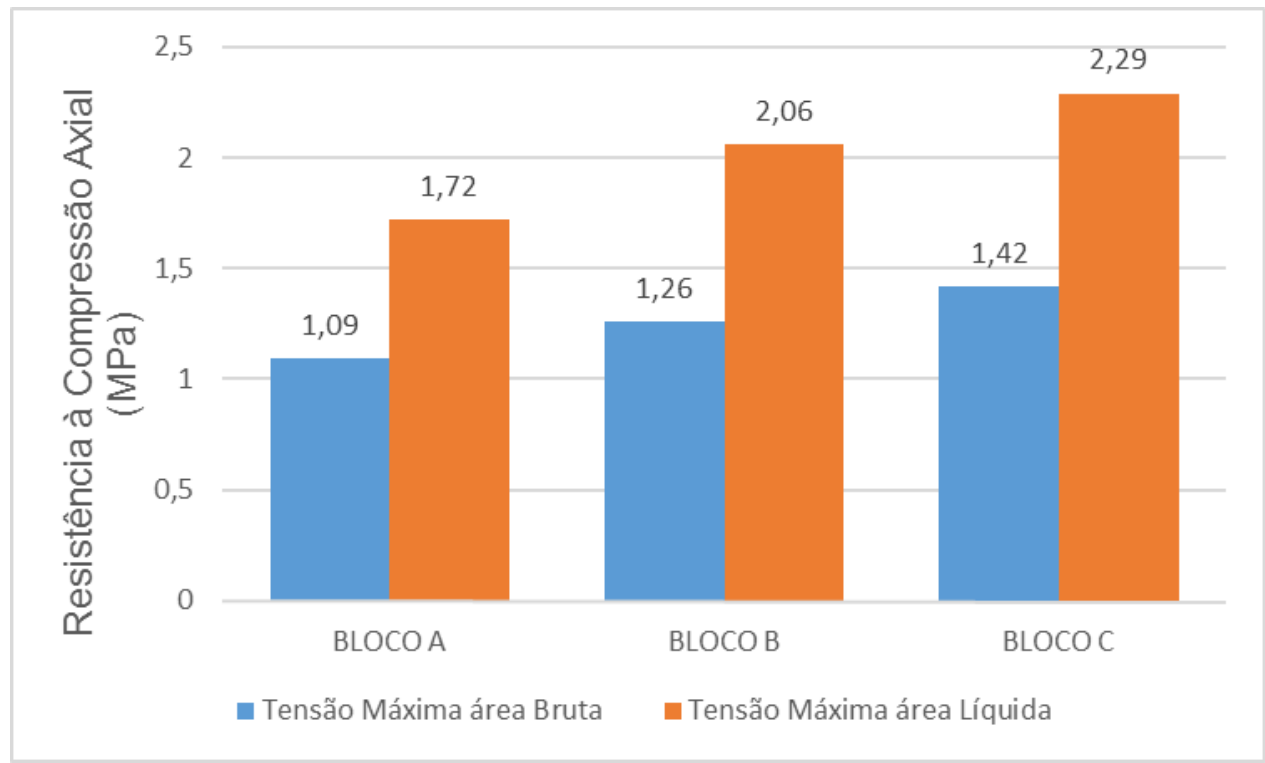

Figura 5. Valores médios de resistência à compressão axial para áreas total (?) e líquida dos blocos. 
Nas comparações dos valores de resistência à compressão axial utilizando as áreas bruta e líquida, observa-se um acréscimo médio em torno de $60 \%$. Portanto, caso os blocos fossem produzidos sem a parte vazada, ou seja, maciços, a resistência seria elevada em torno de $60 \%$, entretanto, isso resultaria em aumento de $58 \%$ no consumo de materiais e peso.

\section{Propriedades térmicas dos blocos}

Na Tabela 4 estão apresentados os valores médios da condutividade térmica para os três tipos de blocos produzidos e alguns tipos de materiais de construção.

Tabela 4. Condutividade térmica dos blocos de cimento-madeira vazados e de alguns materiais de construção.

\begin{tabular}{ccc}
\hline Materiais & $\begin{array}{c}\text { Densidade Aparente } \\
\left(\mathbf{k g} / \mathbf{m}^{\mathbf{3}}\right)\end{array}$ & $\begin{array}{c}\text { Condutividade térmica } \\
\mathbf{( W / m K )}\end{array}$ \\
\hline Bloco A & 540 & 0,190 \\
Bloco B & 720 & 0,217 \\
Bloco C & 690 & 0,234 \\
Concreto normal * & $2.200-2.400$ & 1,75 \\
Concreto pozolônico * & $1.000-1.200$ & 0,35 \\
Placa de gesso * & $750-1.000$ & 0,35 \\
Placa de compensado * & $450-550$ & 0,35 \\
Madeira (pinus, cedro, pinho) * & $600-750$ & 0,23
\end{tabular}

OBS: Valores da tabela * foram obtidos da norma NBR15220 (Associação Brasileira de Normas Técnicas, 2008).

Os valores médios de condutividade térmica dos blocos tipos $A$, B e $C$, variaram de 0,190 W/mK a 0,234 W/mK. De acordo com a NBR 15220 (Associação Brasileira de Normas Técnicas, 2008) baixos valores de condutividade térmica em materiais podem indicar um melhor desempenho térmico nas edificações. Os resultados demonstram que a condutividade térmica elevou-se com o aumento do cimento na mistura, onde os blocos com relação madeira:cimento de 1:2,2 (blocos B e C) apresentaram maiores valores em comparação aos blocos produzidos com relação madeira:cimento de 1:1,5 (bloco A). Todos os blocos produzidos nesta pesquisa apresentaram valores de isolamento térmico superior ao dos materiais cimentícios convencionais e produtos de madeira, apresentados na Tabela 4.

Os materiais vegetais, como a madeira, possuem maior resistividade à condução de calor e, quando incorporados à matriz cimentícia, transferem essas mesmas características ao compósito formado.

$\mathrm{Na}$ análise comparativa com os estudos realizados por outros pesquisadores, os resultados obtidos neta pesquisa, apresentam características de isolamento térmico superior à encontrada por Lima (2009), cujos blocos com cinza de casca de arroz apresentaram valores médios de condutividade térmica de 0,52 W/mK, e, por Stancato (2006), cujos compósitos de Pinus caribaea apresentaram valor médio de $0,68 \mathrm{~W} / \mathrm{mK}$.

\section{CONCLUSÕES}

Os três traços (relação madeira:cimento) utilizados na produção dos blocos apresentaram densidades menores que os blocos convencionais, sendo possível, assim, sua classificação como blocos leves de concreto, de acordo com a NBR 12655 (Associação Brasileira de Normas Técnicas, 2015).

Os blocos produzidos com maior relação madeira:cimento (traço - 1:2,2) apresentaram maior densidade e aumento na resistência à compressão axial.

Os blocos apresentaram reduções significativas de condutividade térmica em comparação a outros produtos cimentícios, demonstrando serem bons isolantes térmicos. 
Os blocos apresentaram resistência à compressão axial menor que o requisito mínimo de 3,0 MPa (NBR 6136, Associação Brasileira de Normas Técnicas, 2014a) estabelecido para bloco estrutural. Entretanto, a sua produção pode ser recomendada para aplicações em alvenaria interna, sem função estrutural, com a substituição de agregado mineral por vegetal (madeira), com consequente redução no peso do produto.

\section{REFERÊNCIAS}

American Society for Testing and Materials - ASTM. (2010). ASTM C 518-10: Standard Test Method for Steady-State Thermal Transmission Properties by Means of the Heat Flow Meter Apparatus. West Conshohocken: ASTM.

Associação Brasileira de Cimento Portland - ABCP. (2013). Básico sobre cimento. Recuperado em 18 de novembro de 2013, de http://www.abcp.org.br/conteudo/basico-sobre-cimento/adicoes/vantagens

Associação Brasileira de Normas Técnicas - ABNT. (2008). NBR 15220: Desempenho térmico de edificações - especificação. Rio de Janeiro: ABNT.

Associação Brasileira de Normas Técnicas - ABNT. (2014a). NBR 6136: Blocos vazados de concreto simples para alvenaria - requisitos. Rio de Janeiro: ABNT.

Associação Brasileira de Normas Técnicas - ABNT. (2014b). NBR 12118: Blocos vazados de concreto simples para alvenaria - métodos de ensaio. Rio de Janeiro: ABNT.

Associação Brasileira de Normas Técnicas - ABNT. (2015). NBR 12655: Concreto de cimento Portland Preparo, controle, recebimento e aceitação - Procedimento. Rio de Janeiro: ABNT.

Castro, V., Parchen, C., \& Iwakiri, S. (2018). Particle size wood/cement ratio effect on the production of vibro-compacted composites. Floresta e Ambiente, 25(4), 1-8. http://dx.doi.org/10.1590/21798087.021315.

Dargahi-Zaboli, M., \& Alizadeh, R. (2014). Using dynamic model for determination of heat losses in cold weather from a typical house in Tabriz constructed by lightweight concrete blocks. Energy Efficiency, 7(4), 609-626. http://dx.doi.org/10.1007/s12053-013-9243-5.

Del Coz Díaz, J. J., García Nieto, P. J., Domínguez Hernández, J., \& Suárez Sánchez, A. (2009). Thermal design optimization of lightweight concrete blocks for internal one-way spanning slabs floors by FEM. Energy and Building, 41(12), 1276-1287. http://dx.doi.org/10.1016/j.enbuild.2009.08.005.

Instituto Carbono Brasil. (2014). Recuperado em 8 de outubro de 2018, de http://www.carbonobrasil.com.br/

Iwakiri, S. (2005). Painéis de madeira reconstituída (243 p.). Curitiba: FUPEF.

Iwakiri, S., Trianoski, R., Fonte, A. P. N., Cezimbra, D. J., Fomin, I. M., \& Molleken, R. (2017). Potencial de uso de espécies de pinus tropicais para produção de painéis cimento-madeira. Scientia Forestalis, 45(113), 1-7. http://dx.doi.org/10.18671/scifor.v45n113.15.

Lima, A. J. M. (2009). Utilização de resíduo de Pinus spp e materiais cimentícios alternativos na produção de blocos para alvenaria estrutural (Tese de doutorado). Universidade Federal do Paraná, Curitiba.

Medeiros, J. S., \& Sabbatini, F. H. (1993). Alvenaria estrutural não armada de blocos de concreto: produção de componentes e parâmetros de projeto (Boletim Técnico). São Paulo: USP.

Nolasco, A. M. (2000). Resíduos da colheita e beneficiamento da caixeta - Tabebuia cassinoides (Lam.) DC: caracterização e perspectivas (Tese de doutorado). Escola de Engenharia de São Carlos, Universidade de São Paulo, São Carlos.

Parchen, C. F. A. (2012). Compósito madeira cimento de baixa densidade produzido com método de compactação vibro dinâmica (Tese de doutorado). Universidade Federal do Paraná, Curitiba.

Parchen, C. F. A., Iwakiri, S., Zeller, F., \& Prata, J. G. (2014). Vibro-dynamic compression processing of low-density wood-cement composites. European Journal of Wood and Wood Products, 74(7), 1-7.

Posi, P., Lertnimoolchai, S., Sata, V., \& Chindaprasirt, P. (2013). Pressed lightweight concrete containing calcined diatomite aggregate. Construction \& Building Materials, 47, 896-901. http://dx.doi.org/10.1016/j.conbuildmat.2013.05.094.

Rech, C. (2002). Estudo sugere uso de serragem como insumo. Revista da Madeira, 66. Recuperado em 8 de outubro de 2018, de http://www.remade.com.br/br/revistadamadeira_materia.php?num=221\&subject=Res\%EDduos\&titl e=Estudo\%20sugere\%20uso\%20de\%20serragem\%20como\%20insumo 
Savastano Junior, H. (2000). Materiais à base de cimento reforçados com fibra vegetal: reciclagem de resíduos para a construção de baixo custo (Tese de livre-docência). Escola Politécnica, Universidade de São Paulo, São Paulo.

Semple, K. E., Evans, P. D., \& Rural Industries Research and Development Corporation. (2004). Woodcement composites - suitability of Western Australian mallee eucalypt, blue gum and melaleucas (RIRDC publication, No. 04/102, 90 p.). Canberra: RIRDC.

Sousa, J. G. G. (2001). Contribuição ao estudo da relação entre propriedades e proporcionamento de blocos de concreto: aplicação ao uso de entulho como agregado reciclado (Dissertação de mestrado). Universidade de Brasília, Brasília.

Stancato, A. C. (2006). Caracterização de compósitos à base de resíduos vegetais e argamassa de cimento modificada pela adição de polímeros para a fabricação de blocos vazados (Tese de doutorado). Universidade Estadual de Campinas, Campinas.

Tango, C. E. S. (1984). Blocos de concreto: dosagem, produção e controle de qualidade (Vol. 1, 110 p.). São Paulo: IPT.

Torkaman, J., Ashori, A., \& Sadr Momtazi, A. (2014). Using wood fiber waste, rice husk ash, and limestone powder waste as cement replacement materials for lightweight concrete blocks. Construction \& Building Materials, 50, 432-436. http://dx.doi.org/10.1016/j.conbuildmat.2013.09.044.

Villas-Bôas, B. T., Parchen, C. F. A., Iwakiri, S., Prata, J. G., \& Costa, M. R. M. M. (2017). Avaliação dos efeitos de diferentes dosagens de água/cal na resistência à compressão axial de compósitos de madeira-cimento. Revista Matéria (Rio de Janeiro), 22(1), e11777. https://doi.org/10.1590/s1517707620170001.0109.

Contribuição dos Autores: BTVB: Conceituação, Curadoria de dados, Investigação, Metodologia, Validação, Escrita - Revisão e Edição; SI: Escrita - Primeira Redação, Escrita - Revisão e Edição; CFAP: Conceituação, Metodologia, Validação. 